Société d'histoire de la révolution de 1848 et des

révolutions du XIXe siècle

$46 \mid 2013$

L'espace du politique en Allemagne au $\mathrm{XIX}^{\mathrm{e}}$ siècle

\title{
Robert Chanaud [dir.], Limousin, terre d'historiens
}

Limoges, Presses universitaires de Limoges, 2012, 282 p. ISBN :

978-2-84287-574-9. 25 euros

\section{Paul D'Hollander}

\section{OpenEdition}

\section{Journals}

Édition électronique

URL : http://journals.openedition.org/rh19/4519

DOI : $10.4000 /$ rh 19.4519

ISSN : $1777-5329$

Éditeur

La Société de 1848

Édition imprimée

Date de publication : 1 juin 2013

Pagination : 235-237

ISSN : 1265-1354

Référence électronique

Paul D'Hollander, «Robert Chanaud [dir.], Limousin, terre d'historiens", Revue d'histoire du XIXe siècle [En ligne], 46 | 2013, mis en ligne le 30 janvier 2014, consulté le 22 septembre 2020. URL : http:// journals.openedition.org/rh19/4519; DOI : https://doi.org/10.4000/rh19.4519

Ce document a été généré automatiquement le 22 septembre 2020.

Tous droits réservés 


\section{Robert Chanaud [dir.], Limousin, terre d'historiens}

Limoges, Presses universitaires de Limoges, 2012, 282 p. ISBN :

978-2-84287-574-9. 25 euros

Paul D'Hollander

\section{RÉFÉRENCE}

Robert chanaud [dir.], Limousin, terre d'historiens, Limoges, Presses universitaires de Limoges, 2012, 282 p. ISBN : 978-2-84287-574-9. 25 euros

1 L'association Rencontre des historiens du Limousin, fondée en 1976 à l'initiative de Louis Pérouas, a publié depuis sa création plusieurs études remarquées, fruit d'une recherche collective de ses membres (de l'ouvrage sur les prénoms en Limousin depuis un millénaire, paru en 1984, à ceux consacrés aux identités limousines ou au paysage et à l'environnement, parus plus récemment). La dernière publication de cette association, aujourd'hui dirigée par Robert Chanaud, contient quinze communications consacrées aux historiens limousins et aux historiens du Limousin. Tous ne sont pas étudiés dans cet ouvrage, et les angles d'observation sont variés, mais les différentes contributions mettent en évidence les «forces qui s'exercent sur l'écriture de l'histoire: déterminismes de l'époque, de la position de l'historien dans le champ historiographique, de sa formation, de ses présupposés, etc. » écrit Robert Chanaud.

Martine Larigauderie-Beijeaud dresse un bilan historiographique sur l'ordre de Grandmont du Moyen Âge à nos jours. La culture historique des moines limousins est présentée par Julien Bellarbre tandis que Jean-Pierre Delhoume montre que l'histoire occupe une place importante dans certaines bibliothèques de Limougeauds au XVIII siècle. Plusieurs figures d'érudits de l'époque moderne sont présentées : le magistrat marchois Pierre Robert par Michel Cassan, Jean-Baptiste Delmas de La Rebière par JeanLoup Lemaître. Deux contributions sont consacrées à certaines lacunes dans l'histoire limousine. Nicole de Blomac déplore que le cheval Limousin, dont au XIX ${ }^{\mathrm{e}}$ siècle, auteurs 
et chroniqueurs louent les qualités, n'ait pas tenté les historiens. Vincent Brousse constate que, dans une terre de gauche, l'histoire des droites en Limousin est un « territoire en friche ». Il compte trois à quatre fois plus de travaux universitaires sur la gauche que sur la droite et pose la question de l'influence du tempérament politique régional sur le champ des études historiques. Plus près de notre époque, Dominique Danthieux montre la difficulté d'écrire l'histoire du drame d'Oradour-sur-Glane.

3 Le « siècle de l'histoire » est très présent dans cet ouvrage collectif. Pour les premières décennies $\mathrm{du}$ XIX $\mathrm{e}^{\mathrm{e}}$ siècle, Philippe Grandcoing analyse la «lecture romantique des pierres " au moment où amateurs et érudits portent intérêt aux restes archéologiques mais sans toujours « savoir séparer le bon grain scientifique de l'ivraie fantaisiste alors dominante » en reprenant souvent ce que d'autres ont affirmé, et en demandant à l'archéologie de corroborer la tradition. Pour cette première moitié du siècle, Agnès Morterol étudie la naissance historiographique des émaux limousins à un moment où il n'y a plus d'émailleur à Limoges, où quelques collectionneurs privés s'intéressent à un art disparu, à ses origines, aux grands foyers de production. Les options politiques des historiens et les polémiques des dernières décennies $\mathrm{du} \mathrm{XIX}^{\mathrm{e}}$ siècle sont abordées par Jean-François Boyer à propos d'une question de géographie historique et par Robert Chanaud qui tente de cerner les opinions des historiens des trois départements du Limousin durant l'âge d'or des sociétés savantes. Dans cette contribution, quelques individualités ressortent (Alfred Leroux, l'abbé Lecler, Louis Guibert, etc.) et l'auteur nous montre combien le choix d'un sujet historique (le meurtre de l'abbé Chabrol à Limoges en 1792 par exemple) pouvait être «un véritable acte de militantisme, parfaitement compris des contemporains, mais où nous ne voyons, le temps ayant passé, qu'une innocente étude érudite ". L'étude de la fondation des sociétés savantes, la place accordée dans leurs bulletins à la Révolution et à l'histoire contemporaine, laissent percevoir la coloration idéologique de chacune. Dans une communication sur "Le Limousin dans l'histoire nationale au XIX ${ }^{\mathrm{e}}$ siècle ", Anne Gérardot a recherché les mentions du Limousin dans un corpus d'histoires générales de la France écrites au XIX siècle (Sismondi, Guizot, Michelet, Lavisse, etc.). Elle se demande si l'on peut " quantifier le "bruit" généré par le Limousin dans l'histoire nationale, appréhender le regard porté sur la province et y distinguer, le cas échéant, des évolutions ». La mémoire collective est-elle aujourd'hui "tributaire» ou non du regard porté sur le Limousin à cette époque? Si les disparités concernant les mentions du Limousin sont importantes, c'est aux XII ${ }^{\mathrm{e}}$-XIV ${ }^{\mathrm{e}}$ siècles que sa présence dans l'histoire nationale est la plus marquée. La période moderne «sans pour autant faire figure de désert historiographique, apparaît relativement terne ». Les notations positives sur Limoges ou le Limousin sont rares. Pour l'époque moderne émergent deux figures: Turgot, occasion pour les historiens de souligner la pauvreté du pays et de ses habitants et l'abbé Dubois, tenu en piètre estime. Les disparités relevées tiennent aux sources utilisées par les différents auteurs (chroniques, archives), à leur volonté parfois Lavisse - de couvrir de manière équilibrée le territoire, d'élargir l'exposé au-delà de l'histoire politico-militaire. Au tournant des $\mathrm{XIX}^{\mathrm{e}}$ et $\mathrm{xx}^{\mathrm{e}}$ siècles, l'histoire de Lavisse, écrite à plusieurs mains, tire profit des travaux des érudits et des sociétés savantes. Une étude sur l'histoire et les activités de l'association Rencontre des historiens du Limousin clôt l'ouvrage.

4 Plusieurs communications permettent de remettre en lumière des amateurs, des érudits aujourd'hui oubliés (Gondinet, Allou, l'abbé Texier, Maurice Ardant, le Comte de 
Seilhac, etc.). Elles montrent pour les premières décennies du XIX ${ }^{e}$ siècle l'importance des initiatives privées, la frilosité parfois des pouvoirs publics. Beaucoup de ces érudits, passionnés d'histoire, sont animés d'un patriotisme local qui les pousse à fouiller le passé sans toujours disposer de grilles de lectures et d'outils efficaces. Claude Petitfrère montre comment la figure du cardinal Dubois, principal ministre du Régent, un "Briviste mal aimé de l'histoire ", tend à être réhabilitée, à partir du milieu du XIX siècle, par des historiens d'abord venus du terroir corrézien "pour lesquels entre en cause ce "patriotisme local", si important dans l'écriture de l'histoire, une affaire d'honneur en quelque sorte». 